\title{
Antithrombin III deficiency and cerebrovascular accidents in young adults
}

\author{
J A Graham, H M Daly, P J Carson
}

\begin{abstract}
A young man with antithrombin III (ATIII) deficiency sustained a cerebellar venous infarct and recovered following treatment with AT-III concentrate. A family study showed that other members were affected. AT-III deficiency in this family was found to be due to a new variant ATIII TRURO 1.

Young patients with strokes should be screened for thrombophilia.
\end{abstract}

(₹ Clin Pathol 1992;45:921-922)

The incidence of cerebrovascular events in young persons $(25-40)$ is uncommon (2 per 10000 per year) ${ }^{1}$ and may be associated with inherited thrombophilia of which the common causes are protein $\mathrm{C}$, protein S, and AT-III deficiencies. Replacement therapy and long term anticoagulation may prevent venous thromboembolism in affected persons.

Department of Haematology, Royal Cornwall Hospital (Treliske), Truro, Cornwall

J A Graham

H M Daly

P J Carson

Correspondence to:

Dr J A Graham,

70 St Ambrose Road, Heath, Cardiff CF4 4BH

Accepted for publication 28 February 1992

\section{Case report}

A 37 year old man (fig 1 (I-IIb)) was admitted with sudden onset of diplopia, dysarthria, and headache followed by collapse. Examination showed slight ataxia and rotatory nystagmus. He was taking warfarin (INR $2 \cdot 2$ ) because an underlying thrombotic defect had been suspected.

\section{Family study}

1

"

(a)

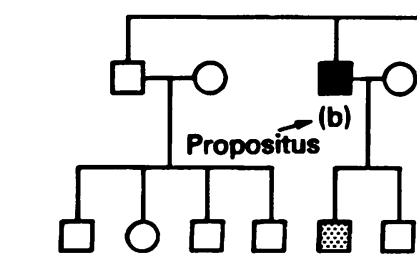

(e)

(g)

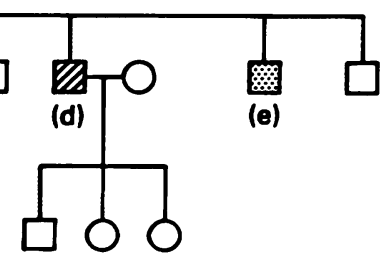

\section{Key:}

- Reduced concemtrations of amtithrombin III and venous thromboembolism

- Normal concentrations of amtithrombin III and no venous thromboembolism - Normal concentrations of antithrombolin III and venous thromboembolism - Normal concentrations of amtithrombolin III and venous thromboembolism
- Reduced concentrations of antithrombin III and no venous thromboembolism

Figure 1 Family tree.

Eighteen months earlier a fractured tibia was complicated by deep vein thrombosis and pulmonary emboli. Up to 80000 units of intravenous heparin were required to achieve satisfactory prolongation of his aplastic prothrombin time (APTT) $(>2 \cdot 0)$ and before achieving this he developed repeated pulmonary emboli, necessitating admission to the intensive care unit.

After admission he became critically ill and developed right cerebellar signs. A computed tomogram of the head (with contrast) (fig 2) showed a low density area in the right cerebellar hemisphere, effacement of the fourth ventricle, and a characteristic "delta" sign (see arrow). These appearances are highly suggestive of cerebellar venous thrombosis. The patient was too ill for angiographic confirmation.

There was a strong family history of venous thrombosis (table). Investigation of the patient for an underlying thrombotic defect showed an AT-III antigen concentration of $66 \%$ (normal range $80-140 \%)$ and showed an activity level of $68 \%$ (normal range $80-130 \%$ ).

Warfarin was discontinued, because of the risk of haemorrhage secondary to the infarct, and heparin withheld because of the risk of a further reduction in AT-III activity. He was treated with AT-III concentrate daily to prevent further venous thrombosis. AT-III concentrations were measured daily and AT-III concentrate was administered to achieve a 20 minute postinfusion level of $100 \%$. The dose was calculated as follows:

$\frac{\text { Dose in Ukg }=\text { desired increase } \times \text { weight }}{1.6 \%}$

(He received 820 units on day 1,820 units 12 hourly on day 2 , and 820 units on day 3 ). He remained seriously ill and for several days his survival was in doubt. However, within one week he began to improve and was given long term warfarin, aiming at an INR of 3.0-4.5. $\mathrm{He}$ was discharged four weeks later and had improved so much that his main concerns were about future employment and ability to drive. $\mathrm{He}$ remains fully active at the time of writing.

\section{Methods}

AT-III activity was measured using the Immunochem AT-III kit (Immuno Ltd) on an ACL 
Figure 2 Computed tomogram showing a low density area in the right cerebellar hemisphere, effacement of the fourth ventricle, and characteristic "delta" sign.

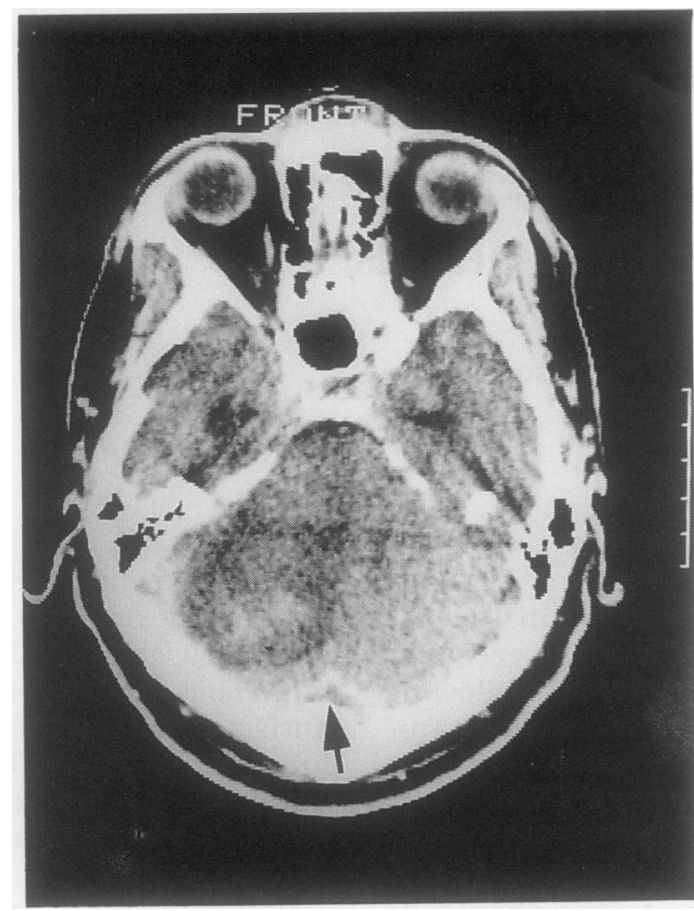

200 analyser (Instrumentation Laboratory UK) and AT-III antigen was measured by immunoelectrophoresis.

\section{Results}

Investigation of the family (fig 1, table) has identified two other members with reduced AT-III concentrations and a history of venous thromboembolism (I(a) and II(c)). In addition II(d) has a history of deep vein thrombosis postoperatively but normal concentrations and II(e) and III(e) have reduced AT-III values but no symptoms.

Further investigation identified a unique mutation in the antithrombin gene of the propositus designated AT-III TRURO $1 .^{2}$ The
History and antithrombin III concentrations of affected family members

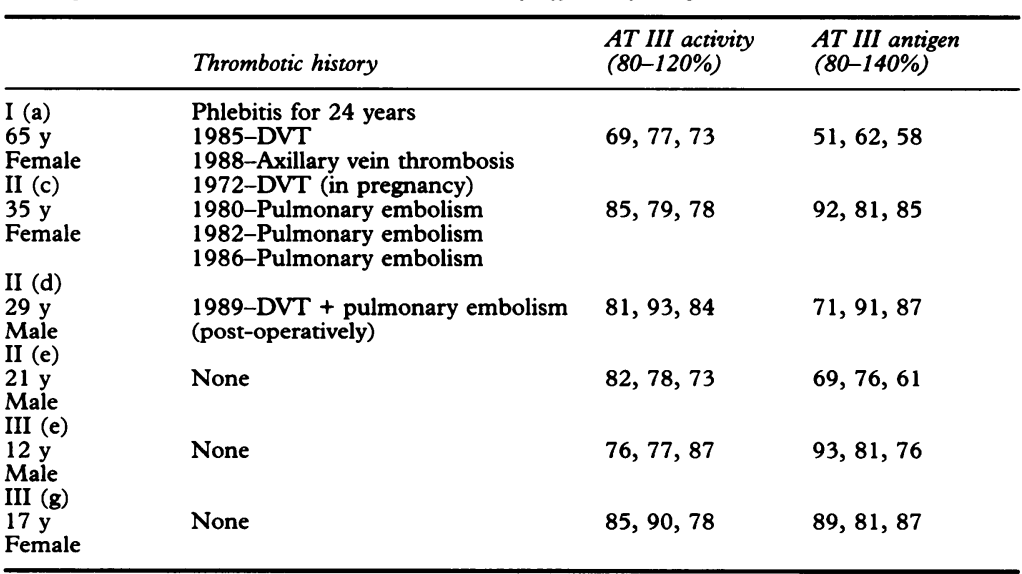

mutation - glutamate $237->$ lysine creates a recognition sequence for the restriction enzyme Xmn 1 permitting rapid screening for the variant. Using PCR and restriction mapping of the amplified DNA I(a), II(c), II(e) and III(g) all have this mutation, but II(d) does not. III(e) has declined further testing.

\section{Discussion}

Inherited AT-III deficiency was first described in $1965^{3}$ and is known to predispose to recurrent premature venous thromboembolism. Inheritance is autosomal dominant with incomplete penetrance. This family exhibits AT-III deficiency with low concentrations of both antigen and bioactivity. Unlike haemophilia when only concentrations of less than $5 \%$ are associated with severe bleeding, AT-III concentrations of less than $80 \%$ may be associated with venous thromboembolism, as in this family. Precipitating factors include pregnancy, surgery, or trauma. By the age of $50,70 \%$ of those affected will have had at least one thrombotic event ${ }^{4}$ which may be fatal.

The propositus was initially difficult to anticoagulate with heparin, a feature of AT-III deficiency, and a clue to the underlying defect.

We report this case to highlight the need to investigate young patients with cerebrovascular events. AT-III is one of the potentially preventable and treatable causes of thromboembolic disease and should be excluded. The propositus was already anticoagulated with warfarin (INR-2.2) when he developed a cerebellar venous infarct, but this proved inadequate. Such patients should be maintained at a higher level with an INR between 3-4.5.

Lifelong treatment with anticoagulants in symptom free AT-III deficient patients is unlikely to improve survival but once they have developed overt thrombosis long term warfarin is advised. ${ }^{5}$ AT-III concentrate is available for treatment of patients with an acute thrombotic event, or prophylactically, when at maximum risk-for example, at the time of delivery or surgery; it reduces the risk of mortality and morbidity.

We are grateful to: Dr I Smith from the Plasma Fractionation Laboratory, Churchill Hospital, Oxford for supplying the Laboratory, Churchill Hospital, Oxford for supplying the Martina Daly and Dr D Perry, Department of Haematology, Martina Daly and Dr D Perry, Department of Haematology,
University of Cambridge, for identifying the mutant antiUniversity of Cambridge, for identifying the mutant anti-
thrombin-III variant; and Dr Robin B Sellwood, Royal Cornthrombin-III variant; and Dr Robin B Sellwood, Royal Corn-
wall Hospital (Treliske), Truro for reporting the computed tomogram.

RCP. Stroke: Towards better management. London: Royal College of Physicians, 1982.

2 Egeberg $O$. Inherited antithrombin deficiency causing thrombophilia. Thromb Diath Haem 1965;13:516-30.

Thaler E, Lechner K. Antithrombin III deficiency and thromboembolism. Clin Haematol 1981;10:369-3.

4 Rosendaal FR, Heijboer H, Briet E, et al. Mortality in hereditary antithrombin III deficiency-1830 to 1989 . Lancet 1991;337:260-1.

5 Winter JH, Fenech A, Ridley A, et al. Familial antithrombin III deficiency. $Q f$ Med 1982;204:373-95. 